\title{
Galvanic Exchange on Reduced Graphene Oxide. Designing a Multifunctional Two- Dimensional Catalyst Assembly
}

\author{
Sachidananda Krishnamurthy ${ }^{1}$ and Prashant V. Kamat ${ }^{2}$ \\ 1. Radiation Laboratory, Department of Chemistry and Biochemistry, University of Notre Dame, Notre \\ Dame, USA \\ 2. Radiation Laboratory, Department of Chemistry and Biochemistry, University of Notre Dame, Notre \\ Dame, USA
}

Two-dimensional graphene based systems have been used in various applications such as energy storage and conversion, photocatalysis, drug delivery etc. The appeal of graphene lies in its large surface area and high electron mobility. Also discovery of Dirac-fermions and the Quantum Hall effect in graphene have added to the interest in this unique material. Graphene oxide (GO) offers a simple and economical route to process single-few layers of graphene sheets with oxygen containing functional groups. The ability of GO to accept and conduct electrons from semiconductors makes it interesting for various applications.

We have succeeded in anchoring semiconducting titanium dioxide $\left(\mathrm{TiO}_{2}\right)$ nano particles and metal nano particles (silver and gold) on the 2-D conductive network of GO. Photogenerated electrons from $\mathrm{TiO}_{2}$ are transported across GO network to reduce silver ions into silver nanoparticles. These silver nanoparticles are then galvanically exchanged with gold ions forming gold nanoparticles. Such interesting chemical transformation on GO surface demonstrates GO's versatile ability to anchor a wide array of nano-particles. This coupled with GO's unique ability to capture and conduct electrons has been used to contrive a two-dimensional catalyst nanomat (Scheme-1). Using methyl viologen as a probe we elucidate the photocatalytic activity of the Semiconductor-GO-Metal nanoassembly and highlight the mediation of GO in charge transfer processes. These findings pave the way for the development of graphene-based multifunctional composites for photo-catalysis and detect and destroy applications.

Transmission electron microscopy helps us support the spectroscopic data of the above chemical transformations (Figure 1). Various data such as particle size, reciprocal lattice spacings, and elemental analysis via energy-dispersive X-ray spectroscopy (EDX) were gathered from TEM. The quantitative analysis of the EDX spectra indicated that the unreacted silver in the sample to be $\sim 25 \%$ and gold to be $\sim 75 \%$ (Figure 2). TEM analysis indicates particles are located at different sites and thus the ability of RGO to facilitate spatially separated deposition of $\mathrm{TiO}_{2}$ and $\mathrm{Ag}$ nanoparticles.

The galvanic exchange process was also monitored through the size distribution of Ag nanoparticles deposited on $\mathrm{RGO}$ before and after the $\mathrm{AuCl}_{4}{ }^{-}$treatment. TEM images used for this analysis are also shown in Figure 3. Reasonably good overlap between the size distribution of $\mathrm{Au}$ with that of $\mathrm{Ag}$ and NPs further gives credence to our arguments made for the galvanic exchange process. The distribution analysis supports the fact that Ag centers act as nucleation sites for Au NPs.

\section{References:}

[1] Above work was published in Journal of Physical Chemistry C, 2013, 117 (1), 571-577.

[2] Authors acknowledge the support by the Office of Basic Energy Sciences of the U.S. DOE through Award DE-FC02-04ER15533 and the University of Wisconsin, MRSEC, NSF Grant DMR-1121288. 


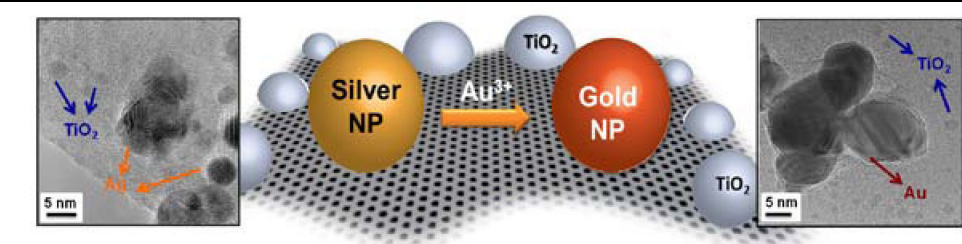

Scheme 1. RGO catalyst mat showing galvanic exchange between silver nano particles and gold ions yielding gold nano particles.

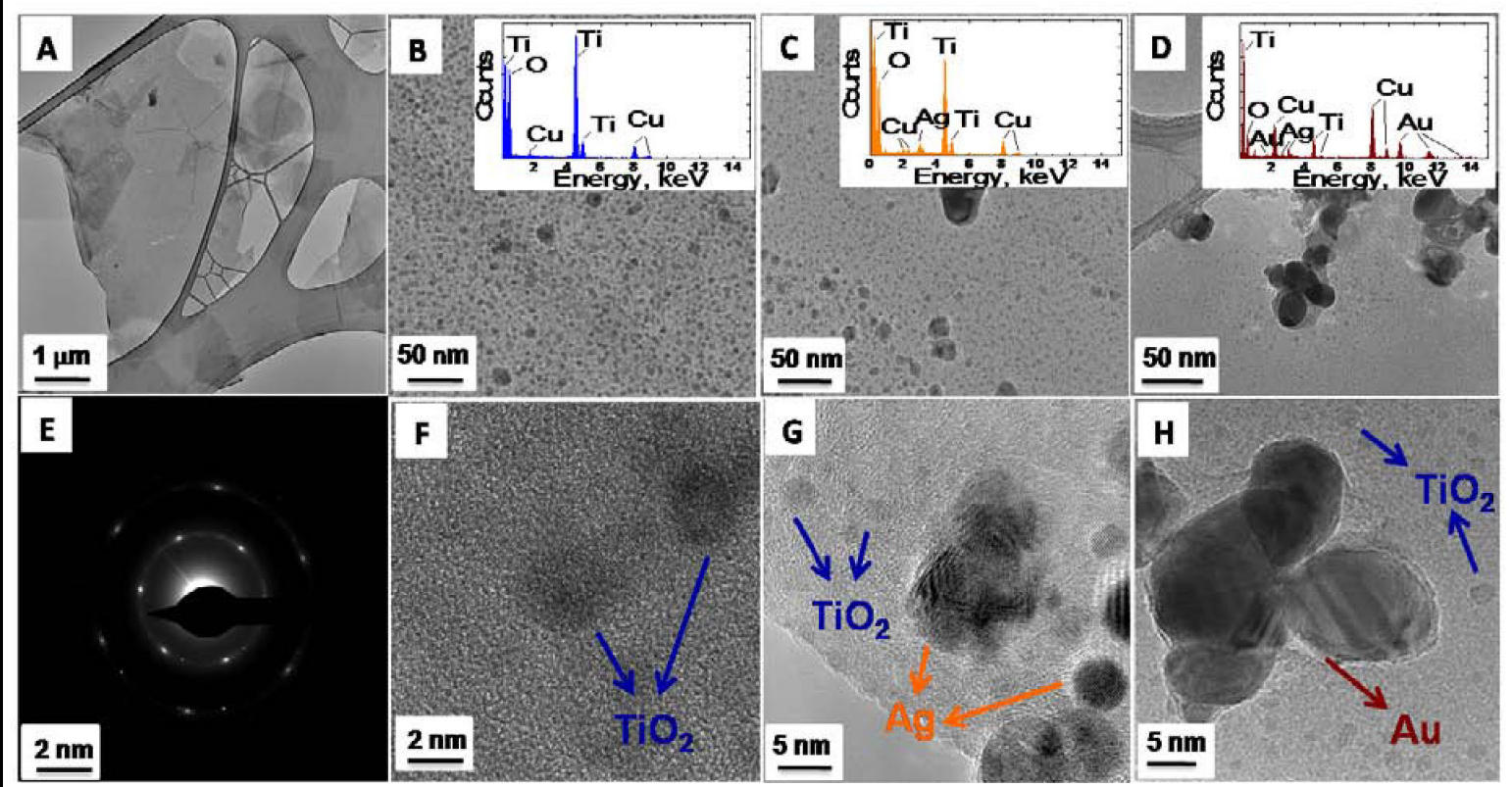

Figure 1. TEM images of (A) Bare RGO sheets, (B) $\mathrm{TiO}_{2}$ particles anchored on RGO sheets, (C) $\mathrm{TiO}$ and $\mathrm{Ag}$ particles and (D) $\mathrm{TiO}_{2}$ and $\mathrm{Au}$ particles anchored on $\mathrm{RGO}$ sheets. Insets of $\mathrm{B}-\mathrm{D}$ show elemental analysis via energy-dispersive X-ray spectroscopy supporting the presence of respective particles on RGO (E) Diffraction pattern of graphene. HRTEM images (F) $\mathrm{TiO}_{2}$ particles anchored on RGO sheets, (G) $\mathrm{TiO}_{2}$ and Ag particles and (H) $\mathrm{TiO}_{2}$ and Au particles anchored on RGO sheets. Samples were prepared by dropcasting $50 \mu 1$ of the respective dispersions and drying over holey carbon grid.

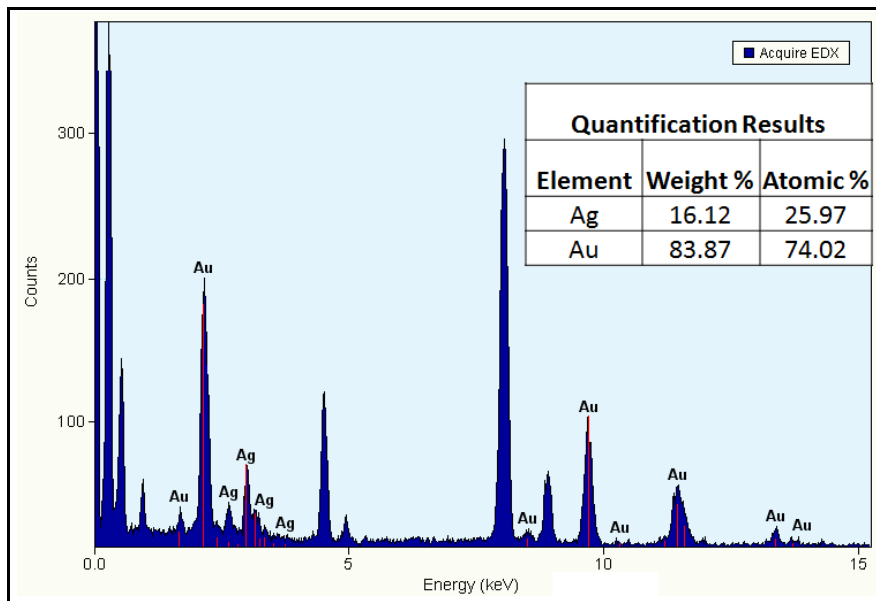

Figure 2. Elemental analysis via energy-dispersive X-ray spectroscopy showing the presence of $\mathrm{Ag}$ and $\mathrm{Au}$ particles on RGO. Inset showing the quantitative distribution of $\mathrm{Ag}$ and $\mathrm{Au}$ elements in the sample.
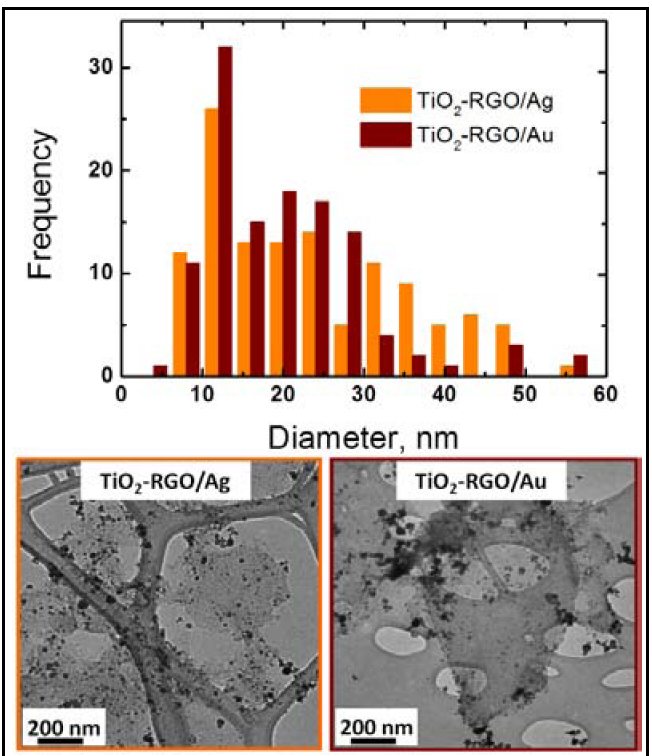

Figure 3. Size distribution of $\mathrm{Ag}$ and $\mathrm{Au}$ NPs on $\mathrm{TiO}_{2}-\mathrm{RGO}$. Also shown are TEM images of $\mathrm{TiO}_{2}-\mathrm{RGO} / \mathrm{Ag}$ and $\mathrm{TiO}_{2}-\mathrm{RGO} / \mathrm{Au}$ composites used for size distribution analysis. 